IdeAs

Idées d'Amériques

$11 \mid 2018$

Modernités dans les Amériques : des avant-gardes à aujourd'hui

\title{
Les années 1968 au Québec
}

1968 in Quebec

1968 en Quebec

Diane Lamoureux

URL : https://journals.openedition.org/ideas/2314

DOI : 10.4000/ideas.2314

ISSN : 1950-5701

Éditeur

Institut des Amériques

Référence électronique

Diane Lamoureux, «Les années 1968 au Québec », IdeAs [En ligne], 11 | 2018, mis en ligne le 18 juin

2018, consulté le 20 octobre 2022. URL : http://journals.openedition.org/ideas/2314 ; DOI : https:// doi.org/10.4000/ideas.2314

Ce document a été généré automatiquement le 20 octobre 2022.



Creative Commons - Attribution - Pas d'Utilisation Commerciale - Pas de Modification 4.0 International - CC BY-NC-ND 4.0

https://creativecommons.org/licenses/by-nc-nd/4.0/ 


\title{
Les années 1968 au Québec
}

\author{
1968 in Quebec \\ 1968 en Quebec \\ Diane Lamoureux
}

1 C'est probablement moins par ce qui s'est passé en France en mai-juin 1968 que par ce qui se déroulait déjà depuis quelques années aux États-Unis, à partir du Free Speech Movement de Berkeley et du rôle du mouvement étudiant dans les mobilisations contre l'intervention étasunienne au Vietnam, que le mouvement étudiant québécois des années 1967-1969 a été marqué, même si Jacques Sauvageot avait été invité à la semaine syndicale de l'UGEQ peu avant la rentrée de 1968 (Lamarre, 2017). Ceci peut s'expliquer, d'une part, par certaines sympathies des militant.es nationalistes québécois pour la figure du général de Gaulle, après que celui-ci a avalisé la cause nationaliste québécoise en criant du balcon de l'Hôtel-de-Ville de Montréal, Vive le Québec libre!. D'autre part, l'identification d'une partie du mouvement nationaliste québécois à des « nègres blancs d'Amérique $»^{1}$ et plus généralement aux luttes menées en Afrique, en Asie et en Amérique latine contre le colonialisme et l'impérialisme, entraînait un intérêt certain à la fois pour le mouvement des droits civiques et pour l'opposition à la guerre du Vietnam.

2 Toujours est-il que le mouvement étudiant québécois entreprend, durant la période 1967-1969, une certaine radicalisation politique, largement imprégnée d'un nationalisme de décolonisation (Mills 2011). À cette époque, la Révolution tranquille, du fait du retour au pouvoir d'un gouvernement de l'Union nationale, présente un certain essoufflement, ce qui amène le mouvement étudiant à quitter sa posture de participation pour une posture plus critique. De plus, les diverses mobilisations sur les enjeux linguistiques, de McGill français aux protestations contre le bill 63, amènent les étudiant.es à jouer un rôle de premier plan dans les mobilisations pour l'indépendance du Québec. Sur le plan organisationnel, on passe d'un syndicalisme, vaguement inspiré de la Charte de Grenoble et de l'UNEF, à des collectifs militants et à l'action directe, parfois liés à des groupes d'extrême-gauche. Mais une chose est certaine : de 1967 à 1969, les étudiant.es sont dans la rue et désertent périodiquement les salles de classe. 


\section{La radicalisation politique en milieu étudiant}

3 Les années 1960 ont été marquées par de profondes transformations dans le milieu de l'éducation au Québec. Jusqu'alors l'éducation, même publique, avait été largement confiée aux communautés religieuses enseignantes. La Révolution tranquille développe une préoccupation majeure pour l'éducation publique, gage de la modernisation du Québec. En 1963 est formée une commission d'enquête sur l'éducation, présidée par le recteur de l'Université Laval, Mgr Parent, commission qui remettra, en 1967, un rapport qui restructurera de fond en comble le milieu de l'éducation au Québec: responsabilité de l'État à tous les niveaux scolaires, curriculum scolaire commun pour les garçons et pour les filles, gratuité scolaire jusqu'à l'université et gel des frais de scolarité à l'université dans la perspective de leur abolition, création d'un réseau préuniversitaire public et gratuit, les Cégeps, dispersés sur une grande partie du territoire québécois.

4 Au début, les associations étudiantes sautent dans le train en marche de la réforme de l'éducation ${ }^{2}$. Cependant, dès 1967, quelques divergences commencent à se manifester. À la rentrée 1968, Cégeps et universités connaissent une certaine effervescence. La cause principale est que la réforme scolaire a largement épargné le niveau universitaire, malgré l'espoir de démocratisation de l'accès aux études universitaires que représentaient les Cégeps. Déjà au printemps 1968, la grogne régnait du fait que 3600 étudiant.es n'avaient pas reçu de soutien financier pour leurs études. En août 1968, l'instance de coordination entre les directions universitaires, la CREPUQ, annonce que plus de 4000 personnes, pourtant admissibles, se verront refuser l'entrée à l'université faute de places et de personnel enseignant dans les universités francophones ${ }^{3}$.

Il n'en fallait pas plus pour déclencher une vague de protestation surtout dans les Cégeps. Peu de temps avant que n'éclatent les premières grèves, les syndicats étudiants avaient décidé de se retirer des comités consultatifs auxquels ils avaient jusqu'alors participé, en invoquant le fait que le gouvernement n'avait ni accordé la gratuité scolaire à l'université, ni converti les prêts en bourses ${ }^{4}$. La majorité des Cégeps seront occupés par les étudiant.es, qui visent ainsi à libérer du temps pour pouvoir discuter de leurs insatisfactions et de leurs revendications. Plus de la moitié des étudiant.es s'attendaient à se voir refuser l'accès à l'université faute de places disponibles en plus de devoir s'endetter du fait de la formule "prêt ", privilégiée par le système d'aide financière aux études. L'annonce, en pleine grève, par le ministre de l'Éducation de l'ouverture d'une deuxième université francophone à Montréal dès la rentrée suivante, n'a pas étouffé la mobilisation et a été reçue avec beaucoup de scepticisme.

Dans la foulée du mouvement d'occupation, d'autres insatisfactions font surface, allant de la méfiance vis-à-vis des syndicats étudiants accusés de complaisance à l'égard des pouvoirs politiques et des administrations du fait de leurs pratiques de concertation et de paternalisme envers la population étudiante. Le caractère essentiellement magistral de l'enseignement allait également être remis en cause. Mais le mouvement étudiant cherchait aussi à sortir des campus et à s'allier aux divers mouvements de contestation des inégalités sociales qui se développaient à la même époque dans les milieux ouvriers et populaires. Une manifestation de 10000 personnes, chiffre assez important pour l'époque, allait marquer l'imaginaire. Mais ce dont le mouvement fait l'expérience en 1968, c'est essentiellement l'action directe et la démocratie des assemblées militantes. 
7 Ces nouveaux savoir-faire militants n'allaient pas tarder à se déployer sur d'autres terrains dans les mois qui suivront : soutien aux chauffeurs de taxi contre le monopole de la compagnie Murray Hill, occupation du centre informatique de Sir George Williams par des étudiant.es noir.es pour protester contre le racisme de certains professeur.es, mais surtout les mobilisations McGill français du printemps 1969 et celles contre le bill 63 à l'automne de la même année.

\section{Les mobilisations autour de la question linguistique}

8 La Révolution tranquille s'accompagne d'une certaine restructuration du mouvement nationaliste. Alors que celui-ci, jusqu'à la fin des années 1950 était marqué par un traditionalisme certain («notre foi, notre langue, nos institutions ») et ne cachait pas ses sympathies pour le pétainisme, au cours des années 1960, il fait de la construction d'un État providence québécois à la fois le lieu privilégié de construction de la cohésion nationale et le fer de lance de la mobilisation pour la souveraineté du Québec. La question linguistique devient le nœud de cristallisation de ce mouvement.

La question linguistique rencontre les préoccupations du mouvement étudiant pour une première fois au printemps 1969, lors de la manifestation McGill français ${ }^{5}$. En effet, à cette époque, il y avait deux universités de langue anglaise à Montréal (McGill et Sir George Williams), tandis qu'il n'y avait qu'une seule université francophone. Le ministre de l'Éducation de l'époque avait eu beau annoncer l'ouverture d'une deuxième université francophone à Montréal afin de calmer les craintes des étudiant.es de ne pas pouvoir accéder à l'université faute de places disponibles, le mécontentement à ce sujet était encore fort palpable et McGill français permettait la jonction de deux cadres contestataires : l'accès aux études universitaires et le statut du français au Québec.

Comme le souligne Arnaud Theurillat-Cloutier, l'enjeu était majeur : «franciser McGill signifiait l'abolition d'un symbole marquant de la domination économique des anglophones sur les francophones» (p. 85). De plus, en pleine guerre du Vietnam, McGill entretenait des liens avec le complexe militaro-industriel étasunien. La manifestation, convoquée par la gauche radicale et les noyaux militants dans le mouvement étudiant, réunit encore plus de monde que la grande manifestation étudiante de l'automne 1968 et donne lieu à plusieurs affrontements avec la police dans les rues du centre-ville de Montréal.

11 Les secteurs les plus radicaux du mouvement étudiant se joignent également aux chauffeurs de taxi, majoritairement francophones, qui protestent contre le monopole de la compagnie Murray Hill sur la prise de voyageurs à l'aéroport de Montréal. Profitant d'une grève des pompiers et des policiers de Montréal, des membres du Mouvement de libération du taxi, appuyés par des sympathisant.es, prennent d'assaut les locaux de la compagnie Murray Hill, incendiant les voitures qui s'y trouvaient. En représailles, les agents de sécurité de la compagnie tirent à balles réelles sur les manifestant.es, tuant une personne et faisant plusieurs blessés.

12 Les affrontements sur la question linguistique reprennent de plus belle à l'automne 1969, lorsque le gouvernement québécois fait adopter, le projet de loi $63^{6}$. Militant.es nationalistes, de gauche et étudiant.es y voient un projet visant à affaiblir l'usage du français au Québec. Là encore, les étudiant.es sont aux premières loges de la mobilisation pour dénoncer ce projet de loi : la grève n'est pas votée partout, mais les 
campus se vident au profit de la rue, le mouvement affectant même plusieurs écoles secondaires. Les manifestations attirent une foule encore plus nombreuse que la manifestation de McGill français: 30000 personnes à Montréal et 20000 à Québec. Malgré l'opposition de la rue, le projet de loi est adopté, ce qui place plusieurs militant.es étudiant.es devant des choix: emprunter la voie institutionnelle en investissant le Parti québécois qui veut poursuivre la Révolution tranquille et faire du Québec un État souverain; se radicaliser du côté des groupes d'extrême-gauche ou du nationalisme radical du Front de libération du Québec; participer à la contre-culture ; se retirer dans le confort de leurs études.

\section{Entre contre-culture, politique et institutions}

13 Après les mobilisations de 1969, le mouvement étudiant reste assez désorganisé et les mobilisations désertent largement les campus. Le cycle de mobilisation ouvert en 1967 prendra fin avec la crise d'octobre 1970, lorsque l'état de siège est proclamé (la loi des mesures de guerre) pour réagir à deux enlèvements de personnalités effectués par des membres du FLQ. Les élections montréalaises de 1970 se déroulent dans un climat de grande polarisation entre un maire qui appuie la répression et qui avait été à l'origine d'un règlement municipal interdisant toute manifestation dans le centre-ville de Montréal après les mobilisations contre le projet de loi 63 et un nouveau parti municipal, le Front d'action politique (FRAP) qui veut profiter des premières élections au suffrage universel ${ }^{7}$ à Montréal pour proposer un programme de justice sociale.

Force cependant est de constater que, à l'instar de ce qui s'est produit ailleurs, les mobilisations étudiantes de la fin des années 1960 n'ont finalement impliquées qu'une minorité d'étudiant.es, même si elles ont produit un effet de génération et un changement de paradigme culturel (Fortin 2006; Warren 2008) . La culture "jeune» s'affirmait et nombre d'institutions sociales, au premier titre desquelles le mariage et la famille, étaient remises en cause.

Après les occupations de 1968, les syndicats étudiants se sont pour la plupart dissous, désertés par les militant.es qui leur ont préféré des structures de mobilisation plus souples, formées des seul.es militant.es. S'il y a une certaine agitation en milieu étudiant qui perdure après les mobilisations de l'automne 1969: expérimentation de nouvelles formes pédagogiques à l'UQAM et dans certains Cégeps; soutien au manifeste du FLQ rendu public suite aux enlèvements d'octobre 1970, par exemple, l'action des secteurs les plus militants se structurera essentiellement autour d'enjeux qui ne concernent pas directement le milieu étudiant: militantisme dans les groupes d'extrême-gauche, solidarité avec certaines grèves ouvrières. Certain.es militant.es vont aussi s'investir dans des causes plus marginales, à l'époque, comme l'écologisme, le féminisme et le mouvement gay.

16 Une partie des étudiant.es qui s'étaient d'abord formés dans le syndicalisme étudiant trouvera dans le Parti québécois un véhicule privilégié pour ses aspirations de modernisation du Québec. Plusieurs étudiant.es feront une longue carrière politique au sein de ce parti.

17 Le mouvement contre-culturel est aussi le produit de ces mobilisations et on en ressent les effets au Québec comme ailleurs en Amérique du Nord. Des revues comme parti-pris tenteront de faire la jonction entre nationalisme radical et contreculture, mais c'est 
surtout sous le label «sex, drugs and rock'n roll » que s'exprimera cette contreculture dont le véhicule principal sera la revue Mainmise.

\section{BIBLIOGRAPHIE}

Fortin, Andrée, Passages de la modernité, Québec, Presses de l'Université Laval, 2006.

Lamarre, Jean, Le mouvement étudiant québécois des années 1960, Québec, Septentrion, 1960.

Mills, Sean, Contester l'empire. Pensée postcoloniale et militantisme politique à Montréal 1963-1972.

Montréal, Hurtubises, 2011.

Theurillat-Cloutier, Arnaud, Printemps de force, Montréal, Lux, 2017.

Warren, Jean-Philippe, Une douce anarchie, Montréal, Boréal. 2008

\section{NOTES}

1. C'est le titre d'un livre de Pierre Vallières, Nègres blancs d'Amérique, (Montréal, Parti-pris, 1968) qui a suscité une certaine gêne lorsque des membres du Front de libération du Québec (FLQ) ont rencontré des membres des Black Panthers à New York à l'été 1968. Signalons également le poème de Michèle Lalonde, Speak white ! qui compare la situation des francophones au Québec à celle des Noirs dans le Sud ségrégationniste.

2. Fortin, Andrée, Passages de la modernité, Québec, Presses de l'Université Laval, 2006.

3. Theurillat-Cloutier, Arnaud, Printemps de force, Montréal, Lux, 2017.

Warren, Jean-Philippe, Une douce anarchie, Montréal, Boréal. 2008.

4. En 2005, ce sera une opération contraire, la conversion de bourses en prêts, qui mettra le feu aux poudres en milieu étudiant.

5. Mobilisation pour souligner les disparités d'accès à l'université entre les francophones et les anglophones à Montréal. Alors que les francophones sont nettement plus nombreux que les anglophones à Montréal, il n'y a qu'une seule université francophone, l'Université de Montréal alors qu'il y a deux universités anglophones (McGill et Sir George Williams). Lorsque les collèges classiques constituaient la seule porte d'entrée à l'université, l'Université de Montréal pouvait admettre la plupart des diplômé.es du collégial. La situation change drastiquement avec la formation des Cégeps en 1967 qui augmente de façon importante le nombre des personnes admissibles à l'Université. L'opération McGill français reçoit des appuis de certains groupes étudiants de l'Université McGill, des groupes étudiants francophones et du mouvement nationaliste québécois

6. Ce projet de loi vise à donner aux parents le choix en ce qui concerne la langue d'enseignement dans le système public québécois. Il est contesté par les groupes nationalistes dans la mesure où il laisse présager une assimilation des populations issues de l'immigration à la minorité anglophone (ce projet de loi avait été motivé par des pressions des organisations italiennes, notamment, qui voulaient que leurs enfants puissent fréquenter l'école anglaise) et d'un certain nombre de francophones étant donné l'attractivité de la langue anglaise sur le continent nord-américain. 
Les enjeux linguistiques restent importants jusqu'en 1977, lorsque le gouvernement du Québec adopte le projet de loi 101 qui fait du français la langue officielle du Québec et oblige tous les enfants (sauf ceux dont les parents ont reçu un enseignement en anglais au Québec) à fréquenter l'école publique française au primaire et au secondaire.

7. Comme le mode de financement des municipalités au Québec est essentiellement la taxe foncière, n'avaient droit de vote que les propriétaires d'un bien immobilier et les locataires en titre, ce qui excluait une grande partie des femmes et bon nombre de membres masculins des couches populaires du droit de vote)

\section{AUTEUR}

\section{DIANE LAMOUREUX}

Professeure associée au Département de Science politique de l'Université Laval, Québec 